\title{
Friedrich Schillers Geschichte des Abfalls der vereinigten Niederlande. Aspekte zum aktuellen Forschungsstand
}

\begin{abstract}
The History of the Secession of the Netherlands from the Spanish Kingdom (1788), the first of Schiller's historical writings, is the starting point for the analysis of the author's position in historical texts within the framework of historiographical studies. This paper attempts to approach the subject from two different perspectives. The first (one) is connected mainly with Schiller's contribution to the methodology of historiography in the period between the Enlightenment and historicism, this approach being inextricably linked with the handling of primary historical sources. The second one concerns the contribution of discourse analysis to the study of Schiller's historical texts, i.e., the application of new instruments to the study of narrative aspects and text composition.
\end{abstract}

Keywords: Schiller; Abfall der vereinigten Niederlande; Historical Thought; Theory on Narrative.

Zusammenfassung: Die Geschichte des Abfalls der vereinigten Niederlande von der spanischen Regierung (1788), die erste von Schillers historischen Schriften, bildet

Der Autor ist Professor für deutsche Literatur und deutsche Kulturgeschichte an der Nationaluniversität Córdoba, Argentinien. 
den Ausgangspunkt für die Untersuchung der Frage, wie sich das wissenschaftliche Interesse an Schiller als Historiker in den vergangenen Jahren artikuliert hat. Dabei nähert sich die vorliegende Arbeit der aktuellen Forschung unter zwei Gesichtspunkten: Zunächst sollen die Beiträge zu Schillers historiographischer Methode vorgestellt werden. Dieser Themenkomplex ist eng mit der Frage der Verwendung historischer Quellen verbunden und hat mit Schillers Position zwischen Aufklärung und Historismus zu tun. In einem zweiten Abschnitt werden erzähltechnische und diskursanalytische Ansätze diskutiert, mit denen in jüngster Zeit Schillers historiographisches Werk untersucht wurde.

Stichwörter: Schiller; Abfall der vereinigten Niederlande; Geschichtsdenken; Erzähltheorie.

Resumo: A "História da Separação dos Países Baixos/da Holanda do Império Espanhol” (1788), o primeiro dos escritos históricos de Schiller, é o ponto de partida para a análise da posição do autor frente a textos históricos no âmbito dos estudos historiográficos. Este artigo tenta abordar o tema de duas perspectivas diferentes. A primeira está ligada principalmente à contribuição de Schiller para a metodologia da historiografia no período entre Iluminismo e Historicismo, estando esta abordagem íntimamente ligada ao tratamento de fontes históricas primárias. A segunda perspectiva diz respeito à contribuição da análise do discurso para o estudo dos textos históricos de Schiller, i. e., à aplicação de novos instrumentos ao estudo de aspectos narrativos e composição do texto.

Palavras-chave: Schiller, Abfall der vereinigten Niederlande, pensamento histórico, teoria da narrativa

Schillers Schaffensperiode zwischen 1787 und 1792, in der alle seine wichtigen historiographischen Werke entstanden sind und auch seine Professur an der Universität Jena liegt, wurde 1995 als "Intermezzo prosaico", als "graue Zone" und "Übergangsphase vom jungen zum späten Dramatiker" etikettiert (DANN 1995: 1). Obwohl sehr schöpferisch und vielseitig, stand Schiller mit seiner historiographischen Produktion immer im Schatten seines dichterischen Werkes. Dafür steht besonders die relativ geringe Beach- 
tung, die sie in der deutschen Germanistik erhalten hat. ${ }^{1}$ Erst seit den 70er Jahren des letzten Jahrhunderts ist von seiten der Geschichtswissenschaft ein neu erwachtes Interesse an den Ansätzen und Methoden der Aufklärungsbewegung und mithin auch an Schiller als Historiker zu verzeichnen. ${ }^{2}$ Ebenso hat die deutsche Literaturwissenschaft, wo längst nicht mehr nur die hochsprachliche Dichtung als ausschließlicher Gegenstand der Disziplin betrachtet wird, den Blick auf den Historiker Schiller ausgeweitet. Unabhängig davon rückte die begriffsgeschichtliche Forschung und das damit verbundene philosophische Interesse die Entwicklung des Geschichtsbewußtseins und der Geschichtstheorie ins Zentrum des Fragens, und auch in diesem Zusammenhang spielte Schillers Geschichtsschreibung eine immer wichtigere Rolle (ibid. 2 f.).

Diese Tendenzen, so kann man in einem Abstand von rund 10 Jahren zu den oben paraphrasierten Befunden sagen, haben sich inzwischen noch erheblich verstärkt. Mein Beitrag möchte am Beispiel von Schillers 1787/ 88 entstandenem historiographischen Erstling Geschichte des Abfalls der vereinigten Niederlande, der auch sein erster großer Publikums- und Verkaufserfolg geworden ist, zeigen, wie sich das wissenschaftliche Interesse an Schiller als Historiker in den vergangenen Jahren artikuliert hat und zu welchen Ergebnissen diese Forschung gelangt ist.

Gliedern möchte ich meinen Beitrag nach folgenden Gesichtspunkten: Zunächst stelle ich einige Ergebnisse der Forschung zur historiographischen Methode Schillers vor, soweit sie an der niederländischen Geschichte demonstriert werden können. Da das Werk in Vorrede und Einleitung eine ganze Reihe programmatischer Äußerungen zu diesem Thema enthält, wird es in diesem Zusammenhang häufig herangezogen. Sodann versuche ich auf geschichtstheoretische, ästhetische und dikursanalytische Ansätze zu verweisen, die in jüngster Zeit an diesem Werk entwickelt wurden. Daraus gewonnene konzeptuelle und begriffsgeschichtliche Erkenntnisse kann ich

Einen kurzen, aber präzisen Überblick über die rezeptions-und wirkungsgeschichtliche Situation gibt die Kölner Diss. von Thomas PrüFer 2000: 3-8.

2 Zum Wandel in der historiographischen Bewertung und Einordnung Schillers zwischen Aufklärungshistorie und Historismus siehe ibid.: 10-18. 
als dritten Punkt aus zeitlichen Gründen hier nicht mehr vortragen. Einschränkend möchte ich bemerken, dass mir die zum Jubiläumsjahr erschienenen einschlägigen Neuerscheinungen leider nicht vorgelegen haben. ${ }^{3}$

Zunächst also zu Schillers historiographischer Methode. Bekanntlich hat der Historismus des 19. Jahrhunderts dem Dichter und Philosophen Schiller jedes Recht auf Anerkennung als Historiker streitig gemacht ${ }^{4}$ zum einen, weil dieser dem Prinzip historischer Quellenkritik angeblich nur ganz ungenügend folgte, zum andern, weil dem Historismus eine theoretische Konstruktion der Geschichte unter dem leitenden, womöglich teleologisch verstandenen Prinzip der Idee, als phantastische Verkürzung bzw. Überformung der Wirklichkeit erschien, die seinem Bemühen um eine, wie er es selbst verstand, unparteiische, auf Entmythologisierung gerichtete und dichterische Verfälschung vermeidende Rekonstruktion der historischen Wahrheit im Wege stand. Zwar gab es schon frühe Versuche zu einer Ehrenrettung Schillers als eines seriös mit seinen Quellen umgehenden Geschichtsschreibers, ${ }^{5}$ doch haben erst Otto Dann und neuerdings vor allem Thomas Prüfer diese Frage in einem breiten Kontext untersucht. Ihre Ergebnisse sollen hier skizziert werden.

Beide Autoren attestieren Schiller, dass er in seinem ersten historiographischen Werk nicht bloß gründliche Auskunft über seine Quellenbasis gibt, sondern auch über Formen und Funktionen historischer Quellen

3 In diesem Zusammenhang hervorzuheben sind Norbert Oellers vielgerühmte Studie Schiller - Elend der Geschichte, Glanz der Kunst (Stuttgart: Ph. Reclam, 2004) sowie Burkhard Müllers Der König hat geweint. Schiller und das Drama der Weltgeschichte (Springe: zu KLAMPEN 2005). Laut Rezension in der Süddeutschen Zeitung vom 12.04.2005 steht im Zentrum dieser Studie eine genaue Betrachtung von Schillers Jenaer Antrittsvorlesung Was heißt und zu welchem Ende studiert man Universalgeschichte?

4 Besonders abschätzig äußerten sich Barthold Georg Niebuhr 1809 und der katholische Historiker Johannes Janssen in seiner 1863 erstmals aufgelegten Studie Schiller als Historiker. Vgl. dazu ausführlich SEEBA 1982: 232-235; PRÜFER 2002: 5.

5 Karl TomascheK, Schiller in seinem Verbältnis zur Wissenschaft. Wien 1862, zit. bei PRÜFER, ibid., und die von Theodor Kükelhaus und Richard Fester im Rahmen zweier Werkausgaben vorgenommenen Untersuchungen der Quellenarbeit Schillers, zit. ibid. 
reflektiert und zumindest ansatzweise über Charakter und Bonität der von ihm verwendeten Quellen Rechenschaft gibt. ${ }^{6}$ Beide heben auf den Schlüsselsatz in der Vorrede zur niederländischen Geschichte ab, wo Schiller diesbezüglich sein Ideal einer guten Geschichtsschreibung formuliert, nämlich Geschichte

[...] aus ihren ersten Quellen und gleichzeitigen Dokumenten zu studieren, sie unabhängig von der Form, in welcher sie mir von dem denkenden Teile meiner Vorgänger überliefert war, neu zu erschaffen und mich dadurch von der Gewalt frei zu machen, welche jeder geistvolle Schriftsteller mehr oder weniger gegen seine Leser ausübt [...]. (IV, 31)

Obwohl sich Schiller im Verlauf seiner historiographischen Arbeit wiederholt enthusiastisch über das "unendliche Feld" des Quellenmaterials äuBerte, hatte er doch von Anfang an keinen Zweifel daran, wie "ungeheuer" die Arbeit des "Materialsammeln[s]" für ihn sein würde. ${ }^{7}$ Und so musste er schließlich auch in der Vorrede eingestehen, dass er nicht "über Armut an Quellen [...] eher über ihren Überfluss" zu klagen habe (IV, 30), zumal "weil man sie alle gelesen haben müsste, um die Klarheit wieder zu gewinnen, die durch das Lesen Vieler in manchen Stücken leidet". Am Ende stellte er deshalb bedauernd fest, seinen Vorsatz nicht einlösen zu können, sonst "hätte aus einem Werke von etlichen Jahren das Werk eines Menschenalters werden müssen". (IV, 31)

Die Befunde zu Schillers historiographischer Arbeitsweise und Verwendung des Materials lassen sich etwa so resümieren:

1. Der von Schiller verwendete Quellenbegriff war zwar umfassend, beschränkte sich jedoch auf sprachliche Überreste. Dabei machte er in seiner Literaturübersicht die bedeutsame Unterscheidung zwischen zeitgenössischer und späterer Überlieferung, und hob deshalb folgerichtig "Memoires", "Briefwechsel”, „Prozeßakten”, “Aktenstücke” und "Bro-

Zum folgenden DanN 1995: 113-120 sowie PrüFER 2002: 278-289.

Briefe an Körner vom 23.2.1788 und Januar 1789, SNA 25, 19 bzw.179, zit. bei DANN 1995: 119. 
schüren jener Zeit" hervor, während er die später entstandene Geschichtsschreibung nur mit den Namen der Autoren bezeichnete (IV, 30f.). Seine Wertschätzung zeitgenössischer Zeugnisse kommt an verschiedenen Stellen des Geschichtswerks zum Ausdruck (z.B. IV, 105, 154 Anm. 2, 210 Anm. 2)

2. Die oben zitierten Formulierung "aus ihren ersten Quellen und gleichzeitigen Dokumenten" deutet darauf hin, dass sich Schiller der heute gängigen Unterscheidung zwischen einer absichtlichen und unabsichtlichen Überlieferung bewusst war, er hat sie aber nicht systematisch entwickelt.

3. Von den insgesamt 29 nachweisbaren Quellen, die er für die Geschichte der niederländischen Rebellion verwendet hat, sind - einschließlich der antiken Autoren, die nur für die kurze Darstellung der alten Geschichte relevant sind - 14 als zeitgenössische Zeugnisse zu bezeichnen. Diese spielen jedoch neben den nicht-zeitgenössischen Darstellungen der Historiker, auf die er sich hauptsächlich stützt, nur eine untergeordnete Rolle.

4. In seiner kritischen Grundhaltung ging es Schiller vor allem um die Wahrheit der Quelle, d.h. die Richtigkeit ihrer Aussage. Damit war die zeitliche, räumliche oder institutionelle Nähe zum Geschehen gemeint, aber auch der Standpunkt des Autors. Wenn nun verschiedene Sichtweisen sich nicht harmonisieren ließen, hat Schiller die Positionen kritisch reflektiert und seine eigene Stellungnahme aus dieser Auseinandersetzung heraus entwickelt und begründet. ${ }^{8}$ In unserem Werk kann die Beschreibung des Bildersturms dafür als exemplarisch gelten, die mit einer Diskussion der verschiedenen Ansichten über die "Triebfedern der außerordentlichen Begebenheit" beginnt und in eine eigenständige Deutung Schillers mündet (IV, 214f.)

5. Im gelehrten Sinn war Schiller sicherlich kein Geschichtsforscher. So hat er selbst keine Archivforschungen getrieben - was damals auch nicht unbedingt zur Tätigkeit des Historikers gehörte - und auch die

8 Vgl. dazu bes. aus der Vorrede: "Bei so ungleichen, relativen, oft ganz widersprechenden Darstellungen derselben Sache hält es überhaupt schon schwer, sich der Wahrheit zu bemächtigen, die in allen teilweise versteckt, in keiner aber ganz und in ihrer reinen Gestalt vorhanden ist." (IV, 30). Als Beispiel für Schillers Diskussion um den Wahrheitsgehalt unterschiedlicher Quellenangaben siehe IV, 164 Anm. 2. 
von ihm benutzten Quellen nicht auf ihre Echtheit geprüft. Die zeitgenössische historisch-philologische Quellenforschung und ihre Gelehrsamkeit hat er zwar gekannt, sie hat ihn aber nicht interessiert. Er selbst war sich seiner Grenzen wohl bewusst. ${ }^{9}$

6. Die Quellen liefern dem Historiker lediglich "Aggregate" von Daten, d.h. einen lückenhaften, fragmentarischer Zusammenhang der Begebenheiten. Seine Aufgabe ist es nun, sie durch kausale Verknüpfungen und analogische Ergänzungen, also durch mentale Verfahren in ein "System" zu bringen. Ein solches "System" konstituiert sich letztlich für ihn aber erst dadurch, dass der Historiker die Abfolge von Begebenheiten teleologisch als finalen Entwicklungszusammenhang deutet und der Geschichte damit einen Sinn gibt. ${ }^{10}$ Bei dieser Operation bleiben die Quellen jedoch immer eine Basis zur Überprüfung der Aussagen und damit ein notwendiges Korrektiv bei der Geschichtsschreibung.

Damit kommen wir zu unserem zweiten Punkt, nämlich den geschichtstheoretischen, ästhetischen und linguistischen Implikationen von Schillers Geschichtsschreibung. Gehen wir auch hier wieder von einem Zitat,

9 In der Hochphase seiner Arbeit an der Geschichte des niederländischen Aufstands schrieb er an Körner: "Auch sehe ich recht gut voraus, dass ich durch meine Arbeit in der Historie, mir einen wesentlicheren Dienst leisten werde, als der Historie selbst, und dem Publikum einen angenehmeren als einen gründlichen den Gelehrten.” Zit. bei PRÜFER 2002: 288; dort auch zitiert sein Bekenntnis gegenüber Caroline von Beulwitz nach Vollendung des Werkes, er "werde immer eine schlechte Quelle für einen künftigen Geschichtsforscher seyn".

10 Theoretisch entfaltet Schiller den Zusammenhang von Aggregat und System erst in seiner Jenaer Antrittsvorlesung (IV, bes. 763f.), doch lässt sich, wie aus dem folgenden hervorgeht, schon an seinem ersten großen Geschichtswerk demonstrieren, wie er selbst in der Praxis damit umgegangen ist, freilich auch seine Schwierigkeiten hatte, die Daten zu verknüpfen und in ein schlüssiges "System" einzubetten. Zur Verwendung und Bedeutung der Begriffe Aggregat und System im geschichtstheoretischen Diskurs der deutschen Spätaufklärung, insbesondere bei den beiden führenden Göttinger Historikern Johann Christoph Gatterer und August Ludwig Schlözer, siehe FuLDA 1996: 155-182, sowie punktuell PRÜFER 2002: 293f. FULDA (ibid.: 231 Anm. 8) sieht bei Schiller allerdings keine direkte Übernahme der beiden Begriffe von den beiden Spätaufklärern. Zu Schillers Systembegriff ibid.: 232f. 
nämlich der Schlussbetrachtung in der Vorrede seiner niederländischen Geschichte aus:

Meine Absicht bei diesem Versuche ist mehr als erreicht, wenn er einen Teil des lesenden Publikums von der Möglichkeit überführt, daß eine Geschichte historisch treu geschrieben sein kann, ohne darum eine Geduldprobe für den Leser zu sein, und wenn er einem andern das Geständnis abgewinnt, daß die Geschichte von einer verwandten Kunst etwas borgen kann, ohne deswegen notwendig zum Roman zu werden. (IV, 31)

Dieser Satz erhält erst vor dem Hintergrund von Schillers intensiver Auseinandersetzung mit Körner und - aus der Retrospektive dieser Diskussion - mit Caroline von Beulwitz über das Verhältnis von Dichtung und Historiographie seine ganze Bedeutung, er reflektiert etwas von Schillers geschichtstheoretischem Konzept. Körner hatte die Debatte mit einem Plädoyer für den Vorrang der Dichtkunst gegenüber der Geschichtsschreibung eröffnet mit dem Argument von der "Undankbarkeit des Geschäfts, Gewißheit zu suchen, wo es an Datis fehlt". Der Historiker finde für die "Opfer die er der Wahrheit zu bringen glaubt", keine Entschädigung. Dem "Romanschreiber" stehe dagegen "ein Reichthum von unter-haltenden Gemählden aus der wirklichen Welt" zur Verfügung - "und eine solche Gallerie ist ein Kunstwerk von größerem Gehalt, als die meisterhafteste Geschichte. Der Vorzug der Wahrheit ist die Täuschung. Wird nicht jede Geschichte durch lebhafte Darstellung zum Roman?’"11 Und wenig später fordert er Schiller direkt dazu auf, die Geschichte nur als Mit-tel für die Dichtkunst zu verwenden: "Wenige historische Data sind hinreichend ein neues Ideal in Deiner Seele zu erzeugen, indem Du das Fehlende durch Phantasie ergänzest [...] Als Dichter hast Du Sprache, Kunstfertigkeit, Phantasie vor tausenden voraus. Als Ge-schichtsschreiber stehst Du tausenden in allem nach, was vieljähriges Studium erfordert." ${ }^{\prime 2}$

In seiner Antwort wehrte Schiller Körners "Geringschätzung der Geschichte" ab und zeigte, worauf es ihm ankam: Auch er stellte die innere Wahrheit der Dichtung über die äußere der Geschichte, doch wollte er nicht

11 Körner an Schiller, 4. 1.1788, NA 33 I, 163, zit. bei PRÜFER 2002: 142.

12 Körner an Schiller, 21.1.1788, NA 33 I 166, zit. ibid.: 143 f. 
wie Körner Geschichte in Kunst auflösen, sondern für die Geschichtsschreibung eine kunstvolle Form finden, die aber deren Eigenständigkeit als empirischphilosophische Wissenschaft wahrte. Auf jeden Fall sollte, wie er in der Vorrede schrieb, dabei Geschichte "nicht zum Roman werden". Und er ging noch einen Schritt weiter, wenn er "aus eigenen Erfahrungen" sagte, "dass die uneingeschränkteste Freiheit, in Ansehung des Stoffes, die Wahl schwerer und verwickelter macht, daß die Erfindungen unserer Imagination bei weitem nicht die Autorität und den Credit bei uns gewinnen, um einen dauernden Grundstein zu einem solchen Gebäude abzugeben, welche uns Fakta geben, die eine höhere Hand uns gleichsam ehrwürdig gemacht hat". Bei beiden aber, bei der Geschichtsschreibung und bei der Dichtung, ist die "philosophische innere Nothwendigkeit [...] gleich". Dabei übersah er durchaus nicht die Schwierigkeiten, um diese innere Notwendigkeit aufzuspüren und umzusetzen, denn in der Tat schien ihm die Geschichte als "willkührlich, voll Lücken und oft sehr unfruchtbar, aber eben das willkührliche in ihr könnte einen philosophischen Geist reitzen, sie zu beherrschen, das leere und unfruchtbare einen schöpferischen Kopf herausfodern, sie zu befruchten und auf diese Gerippe Nerven und Muskeln zu tragen."13

Umgekehrt verteidigte Schiller gegenüber Caroline von Beulwitz den Wahrheitsanspruch der Dichtung, nachdem diese der Geschichte den "Vorzug der Wahrheit" gegeben hatte. Schiller fragte,

ob die innre Wahrheit, die ich die philosophische und Kunstwahrheit nennen will, und welche in ihrer ganzen Fülle im Roman oder in einer andern poëtischen Darstellung herrschen muß, nicht eben so viel Werth hat als die historische [...] Die innre Uebereinstimmung der Wahrheit wird gefühlt und eingestanden, ohne daß die Begebenheit wirklich vorgefallen seyn muß. Der Nutzen ist unverkennbar. Man lernt auf diesem Weg den Menschen und nicht den Menschen kennen, die Gattung und nicht das sich so leicht verlierende Individuum: in diesem großen Felde ist der Dichter Herr und Meister; aber gerade der Geschichtschreiber ist oft in den Fall gesetzt diese wichtigere Art von Wahrheit seiner historischen Richtigkeit

13 Schiller an Körner, 7.1.1788, NA 25, 2, zit. ibid. 144f. und Süssmann 2002: 79 f. 
nachzusetzen, oder mit einer gewissen Unbehilflichkeit anzupaßen, welches noch schlimmer ist. ${ }^{14}$

Auch hier sollen resümierend einige Schlüsse referiert werden, die die neuere Forschung aus diesen Positionen gezogen hat.

1. Die Ästhetisierung der Wissenschaft betrachtete Schiller als die eigentliche Leistung des "schöpferischen Kopfes". ${ }^{15}$

2. In seinen Reflexionen über das Verhältnis von Geschichtswissenschaft und Kunst entwickelte Schiller die Vorstellung von einer Einheit der beiden in ihrer Verschiedenheit. Was beide verbindet, ist ihre 'Konstruktivität', die auf der schöpferischen Einbildungskraft ${ }^{16}$ des 'Genies' beruht. Den Begriff 'Genie' wendet Schiller auf den Künstler und den schöpferischen Historiker gleichermaßen an. Beiden geht es im Gegensatz zur äußeren Wahrheit des Faktischen um "innre Wahrheit", also um einen geistigen Gehalt. Dabei handelt es sich um eine Form innerer Kohärenz, die die Gestalt eines harmonischen Ganzen annimmt, wo Wahrheit und Schönheit eins sind. ${ }^{17}$

14 Schiller an Caroline von Beulwitz, 10.12.1788, NA 25, 154, auszugsweise zit. bei KoOPMANN 1995: 70. Prüfer (2002: 153f.) hält trotz vermeintlicher Gegensätze zu früheren Aussagen Schillers gegenüber Körner daran fest, dass sich das historiographische Programm im Kern nicht geändert, lediglich in einem unterschiedlichen Schreib- und Lebenskontext der Argumentationsschwerpunkt verschoben hat.

15 Vgl. auch die entsprechende Passage aus dem oben zit. Brief an Körner vom 7.1.1788 (NA 25, 2f.), der mit dem Beginn seiner Arbeit an der niederländischen Geschichte zusammenfällt: "Wenn es eine Nothdurft ist, die Geschichte zu lernen, so hat derjenige nicht für den Undank gearbeitet, der sie aus einer trockenen Wissenschaft in eine reitzende verwandelt, und da Genüsse hinstreut, wo man sich hätte gefallen lasssen müssen, nur Mühe zu finden.” Zit. bei PRÜFER 2002: 123.

16 SÜssmann (2000: 77-79, 83f.) sieht in der Einbildungskraft die herausragende Qualität des philosophischen Kopfes, die an die Stelle der "Autopsie" tritt und den epochalen Schritt von der untergegangenen objektivistischen zur neuen subjektivistischen Geschichtsschreibung markiert.

17 PRÜFER, 2002: 135f. Zur Gleichsetzung von Künstler und Wissenschaftler als Genie und zum Ziel von dessen Tätigkeit, die nicht eher ruht, "bis alle seine Begriffe zu einem harmonischen Ganzen sich geordnet haben", siehe Schillers Jenaer Antrittsrede IV, 752. Zum Wahrheitsbegriff vgl. auch Koopmann, 1995, 71-76. 
3. In engem Zusammenhang mit diesem Punkt ist als Gemeinsames von Dichtung und Geschichte die philosophische Form, die Perspektivierung zu sehen, die den fragmentarischen und kontingenten Daten der Geschichte und den imaginierten Handlungen der Dichtung einen Zusammenhang verschafft, so dass sie verstehbar werden. Historische wie poetische Erzählung erhalten auf diese Weise einen Plot, der sie zu einem in sich sinnvollen, sich selbst erklärenden Ganzen macht. ${ }^{18}$ Auf diesen Punkt muss gleich noch näher eingegangen werden.

4. Bei diesem Bemühen um eine narrative Plotstruktur seiner Geschichte entwickelte Schiller einen neuen zukunftsweisenden Wahrheitsbegriff: der genialische Sinn für das Ganze führte ihn zu einer nicht-antiquarischen, interpretatorischen Forschung und Deutung der Geschichte, bei deren Verwirklichung empirische Seriosität und stilistische Eleganz miteinander verbunden werden sollen. In diesem Sinn verstand er das Ideal seiner Geschichtsschreibung als Synthese empirischer, philosophischer und poetisch er Verfahren, durch die er die trockene, lückenhafte und oft willkürliche Geschichte mit 'dichterischem Geist' erfüllen wollte. Zentrales Moment dieser Synthese ist die oben genannte 'Konstruktivität', die ihren Grund in der Tätigkeit des Genies hat. ${ }^{19}$

Verweilen wir kurz bei der Frage, wieweit Schillers narrative Verfahren eine Plotstruktur der Geschichte hervorbringen - letztlich natürlich eine Fragestellung, die mit dem 'linguistic' bzw. 'narrative turn' in den Geisteswissenschaften verbunden ist und im Zentrum der Arbeiten von Daniel Fulda und Johannes Süssmann zur Historiographie Schillers steht. Fulda versucht, wie andere schon vor ihm, ${ }^{20}$ Schiller zwischen den beiden

18 Fulda, 1996: 254f.; dazu kritisch Süssmann, 2000: 31, 80, 84ff. Prüfer, 2002: 19, 145f., der die Reichweite von Fuldas Ansatz anders einschätzt und seinen Beitrag positiv hervorhebt.

19 PRÜFER, 2002: 145f., 154, mit einem kurzen Abriss zum Transformationsprozeß des rhetorischen Erzählbegriffs in einen narrativistischen, bei dem nach seiner Auffassung Schillers Historik eine entscheidende Rolle gespielt hat. Vgl. auch Süssmann, 2000: 110.

20 Die Diskussion darüber beginnt m.W. bei Friedrich MeINECKE (1965: 287 f.). Wichtige Beiträge dazu stammen von Jörn RüsEN (1993) und Ulrich MuHLACK 
Paradigmen "Aufklärungshistorie" und "Historismus" geschichtstheoretisch $\mathrm{zu}$ verorten, und kommt zu dem Ergebnis, dass er darum vielleicht "am wenigsten ein 'moderner' Geschichtsschreiber ist, insofern er in seinen historiographischen Werken immer wieder exemplarische Sinn-bildung betreibt. [...] Das vermögen auch die Ansätze zur Verfabelung seiner Geschichtswerke nicht vollständig zu kompensieren.” (FuLDA 1996: 251) Exemplarische Sinnbildung - im Gegensatz zur genetischen Sinnbildung zeichnet sich für Fulda dadurch aus, dass sie sich weder auf das Verlaufsmoment von (monographischen) Geschichten stützt noch in erster Linie auf die Eigenheiten von deren Fabeln rekurriert. Stattdessen sucht sie das Vorbildliche (oder Abschreckende) von seinem kontingenten Handlungskontext zu befreien und auf historisch invariante Prinzipien zurückzuführen. Besonders stark präsent sei diese Art der Sinnbildung noch in Schillers niederländischer Geschichte auf Grund ihrer eigentümlichen Struktur: "Vergangenheit und Gegenwart können unter exemplarisch verwirklichten moralischen und politischen Prinzipien immer wieder explizit kurzgeschlossen werden, wohingegen eine genetische Sinnbildung in die Makrostruktur des ganzen Textes eingesenkt" sei (ibid.: 253). Damit stellt Fulda die Frage nach dem 'plot' dieser Monographie, der in Konkurrenz zur punktuell betriebenen exemplarischen Sinnbildung die genetische zu tragen habe. Und er stellt fest, dass die niederländische Geschichte, von dessen sechs geplanten Bänden nur einer erschien, "keinen einheitgebenden Plot" besitze, wenngleich zu erkennen sei, dass Schiller sich um eine literaturanaloge Strukturierung des historiographischen Textes bemüht habe. Eine Fabel des Ganzen fehle jedoch, nur einzelnen Teilen - etwa der Geschichte der Geusen im dritten Buch, ${ }^{21}$ die freilich nicht in die von Schillers eingangs eröffnete Begriffsopposition 'Freiheit' und 'Herrschsucht'

(1995), die hier aus Platzgründen nicht besprochen werden können. Einen guten Forschungsüberblick liefert PRÜFER 2002: 12-17, der in seiner Arbeit auf die Anwendung einer paradigmatischen Unterscheidung von "Aufklärungshistorie" und "Historismus" bewusst verzichtet.

21 Fulda nennt auch die geschlossene Form des Kapitels "Bürgerlicher Krieg" als Beispiel für eine gelungene Verfabelung des Stoffes unterhalb der Makrostruktur des gesamten Textes. Fulda 1996: 255 Anm. 8. 
passe und auch andere Inkonsistenzen aufweise ${ }^{22}$ - könne eine Plotstruktur zugesprochen werden (ibid.: 254 f.). Sein Fazit: Die Tatsache, dass sich in den historischen Schriften wenn nicht schon voll-, so doch "teilintegrative Plotstrukturen" finden, mache Schillers Geschichtsschreibung zu einer "Vorform des modern-narrativen Typs". Damit vermittle er zwischen Aufklärungshistorie und historistischer Geschichtsschreibung (ibid.: 260).

Süssmanns Analyse von Schillers Geschichtsschreibung kommt zu etwas anderen Ergebnissen. ${ }^{23}$ Auch er sieht das ganz Neue bei Schiller in dessen Verwendung der historischen "Einbildungskraft", die eine neue Gestalt schafft. Diese Gestalt, die aus einer Synthese besteht, ist dadurch gekennzeichnet, dass sie weder ganz aus Vernunftprinzipien deduziert noch allein aus empirischen Daten abstrahiert, sondern nur mit Hilfe dieser Einbildungskraft zur Anschauung gebracht - und das heißt bei Schiller noch selbstverständlich - erzählt werden kann. Der Historiker habe nach Schiller so zu erzählen, dass das historisch Richtige als etwas Notwendiges erscheint. Nicht indem er zusammenlaufende Kausalketten nachzeichnet, erzeugt er diese Notwendigkeit, sondern "wie im Roman oder einer andern poëtischen Darstellung" durch die Logik der narrativen Verknüpfung. Zwischen historischer Richtigkeit und angestrebter Notwendigkeit existiert eine dauernde Spannung, die sich bei Schiller in Begriffspaaren wie "Stoff und Form", "willkürlichen Bruchstücken und notwendigem Zusammenhang", "Gerippe und Gestalt” äußert. (Süssmann 2000: 78-80, 83-85)

22 So etwa die Tatsache, dass der als Führer der Freiheit schon vorgängig inthronisierte Wilhelm von Oranien nicht auf der Seite der Geusen, sondern der Statthalterin Margaretha steht, und die Geusen selbst, die als vorbildlich angekündigt werden, auf grund ihrer sittlichen Mangelhaftigkeit scheitern. Ein anderes Indiz dafür, dass exemplarische und genetische Sinnstruktur miteinander in Konflikt geraten, zeigt sich laut Fulda darin, dass die exponierte Figurenkonstellation nur relativ schwach für die Verfabelung der Geschichte wirksam wird. (FULDA 1996: 255) Eindeutig dem Typus der exemplarischen Sinnbildung verhaftet seien Verweise auf Helden und Kasus der Antike, denen solche des niederländischen Aufstands gleichkommen. Ibid.: $252 \mathrm{f}$.

23 Auf Süssmanns grundlegende Kritik an Fuldas Ansatz, der den allgemeinen geschichtslogischen Erzählbegriff auf den poetologischen der "literarischen Verfabelung" reduziere und nicht unterscheide zwischen Chronik und Fabel bzw. story und plot, kann hier nicht eingegangen werden. Vgl. Süssmann 2000: 31 Anm. 29. 
Vermittels einer Strukturanalyse des Abfalls der Niederlande macht Süssmann in einer langen, hier nicht nachzuzeichnenden Reihe von Argumenten deutlich, dass der Text selbst, je nachdem wie man ihn betrachtet, einmal als Bruchstück, das andere Mal als durchgeführtes Ganzes erscheint. Die Fabel, die Schiller erzählt, "handelt von der Entstehung und dem Scheitern einer Rebellion. Sie schildert die Bedingungen, unter denen eine Opposition so erstarkt, dass sie einen Herrscher zum Zurückweichen veranlasst und sie erzählt, warum sie dann trotzdem untergeht. Sie bietet ein Lehrstück in politischer Organisation", die "fast den Charakter einer Parabel" besitzt (ibid.: 92). So entpuppt sich laut Süssmann die "innere Notwendigkeit" als die Handlungseinheit einer geschlossenen Fabel, der gegenüber die stoffliche Unabgeschlossenheit des Werkes äußerlich bleibt (ibid.: 97). Andere Elemente hingegen, insbesondere die Erzählinstanz, seien durch die "hohe Kunst äußerster Ambivalenz" gekennzeichnet, die dem Erzählten jede Eindeutigkeit nähmen (Ibid.: 104). Schillers Geschichte bleibe an die Darstellung gebunden, ja sie sei wesentlich Darstellung. Nach Süssmann machten aber die literarischen Elemente das Werk so wenig zur Dichtung, wie die historische Richtigkeit ihm den Status einer wissenschaftlichen Arbeit verleihe. Vielmehr begründe es zwischen diesen Extremen in einer Art nachholenden Literarisierung ein in Deutschland so vorher nicht vorhandenes Drittes: die rhetorisch-literarische Geschichtsschreibung mit einer kulturpolitischen Funktion. Diese Geschichtsschreibung wende sich laut Süssmann an die noch junge deutsche Öffentlichkeit - eine allgemeine Öffentlichkeit, deutschsprachig, kulturnational, literarisch sich verständigend. Sie wollte diese Öffentlichkeit befördern, die Kulturnation in ihrer "Einheit", ihrem Zusammenhalt stärken. Und Schiller literarisiere die Historie, weil er dieses kulturpolitische Anliegen mit ihr verfolge. Daher die Rhetorik, der Parabelcharakter, die Moralität seiner Historiographie. Schillers kulturpolitisches Engagement - und das gehöre zu den Verdiensten seiner niederländischen Geschichte - führe die Geschichtsschreibung heraus aus dem akademischen Ghetto vor die Augen der im Entstehen begriffenen deutschen Nation (ibid.: 111). 


\section{Literaturverzeichnis}

SCHILLER, Friedrich. Sämtliche Werke. Bd. IV: Historische Schriften. 3. Aufl. München, Hanser 1962. (im Text zit. als IV unter Angabe der Seitenzahl)

SCHILlER, Friedrich. Werke. Nationalausgabe, begründet v. Julius Petersen, fortgeführt v. Lieselotte Blumenthal u. Benno von Wiese, ed. Nobert Oellers und Siegfried Seidel. Weimar 1943 ff. (im Text zit. mit der Sigle NA unter Angabe von Band und Seitenzahl)

Dann, Otto/ Oellers, Norbert/ Oesterkamp, Ernst (Hrsg.). Schiller als Historiker. Stuttgart, Weimar, Metzler 1995

Dann, Otto, "Schiller, der Historiker und die Quellen". :In: Dann, Otto/ Oellers, Norbert/ Oesterkamp, Ernst (Hrsg.). Schiller als Historiker. Stuttgart, Weimar, Metzler 1995, 109-126.

Fulda, Daniel. Wissenschaft aus Kunst. Die Entstehung der modernen deutschen Geschichtsschreibung 1760-1860. Berlin, New York, Walter de Gruyter 1996.

Koopmann, Helmut. "Das Rad der Geschichte. Schiller und die Überwindung der aufgeklärten Geschichtsphilosophie”. In: DANN, Otto/ OELLERS, Norbert/ Oesterkamp, Ernst (Hrsg.). Schiller als Historiker. Stuttgart/ Weimar, Metzler 1995, 59-76.

MÜHLACK, Ulrich. "Schillers Konzept der Universalgeschichte zwischen Aufklärung und Historismus". In: Dann, Otto/ Oellers, Norbert/ Oesterkamp, Ernst (Hrsg.). Schiller als Historiker. Stuttgart, Weimar, Metzler 1995, 5-28.

PRÜFER, Thomas. Die Bildung der Geschichte. Friedrich Schiller und die Anfänge der modernen Geschichtswissenschaft. Köln/ Weimar/ Wien, Böhlau, 2002. (Beiträge zur Geschichtskultur, Bd. 24)

RÜSEN, Jörn. "Im Vorspiel der Aufklärung. Bürgerliche Identität zwischen Geschichtsbewußtsein und Utopie bei Friedrich Schiller”. In: Jörn RüsEN (Hrsg.). Konfigurationen des Historismus. Studien zur deutschen Wissenschaftskultur. Frankfurt a.M., Suhrkamp 1993, 139-156.

SEEBA, Hinrich C. "Historiographischer Idealismus? Fragen zu Schillers Geschichtsbild”. In: WiTTKOWsKI, Wolfgang (Hrsg.). Friedrich Schiller. Kunst, 
조을 $\quad$ Humanität und Politik in der späten Aufklärung. Ein Symposium. Tübingen, = Max Niemeyer 1982, 229-251.

I Süssmann, Johannes. Geschichtsschreibung oder Roman? Zur Konstitutionslogik von Q Geschichtserzählungen zwischen Schiller und Ranke (1780-1824). Stuttgart, Franz 융. Steiner 2000. (Frankfurter Historische Abhandlungen, Bd. 41) 